Portland State University

PDXScholar

\title{
The Effects of Mercaptocarborane-Capped Gold Nanoparticles on Water Transport Across Skeletal Muscle Sarcoplasmic Reticulum
}

Jerry K. Bohlen Jr.

Portland State University

Follow this and additional works at: https://pdxscholar.library.pdx.edu/honorstheses

Let us know how access to this document benefits you.

Recommended Citation

Bohlen, Jerry K. Jr., "The Effects of Mercaptocarborane-Capped Gold Nanoparticles on Water Transport Across Skeletal Muscle Sarcoplasmic Reticulum" (2017). University Honors Theses. Paper 379.

https://doi.org/10.15760/honors.377

This Thesis is brought to you for free and open access. It has been accepted for inclusion in University Honors Theses by an authorized administrator of PDXScholar. Please contact us if we can make this document more accessible: pdxscholar@pdx.edu. 


\title{
The Effects of Mercaptocarborane-capped Gold Nanoparticles on Water Transport across Skeletal Muscle Sarcoplasmic Reticulum
}

\author{
by \\ Jerry Bohlen \\ An undergraduate honors thesis submitted in partial fulfillment of the \\ requirements for the degree of \\ Bachelor of Science \\ in \\ University Honors \\ and \\ Biology
}

Thesis Adviser

Dr. Jonathon Abramson

Portland State University 


\begin{abstract}
:
This thesis investigated the effects of mercaptocarborane-capped gold nanoparticles on water transport across skeletal muscle sarcoplasmic reticulum (SR). Data suggest that as the concentration of these gold nanoparticles increase, they cause a decrease in the initial rate of water transport, a decrease in the amount of water released from SR vesicles, and a decrease in the rate constant associated with water transport across the SR. Gold nanoparticles likely produce these effects by altering the behavior of the lipids on the SR membrane, causing them to be less permeable to water transport. The mechanism behind this isn't known and would be an interesting subject to investigate further.
\end{abstract}




\section{Introduction:}

Much research has focused on unraveling the mysteries behind muscle physiology. Even though this is the case, there is still a surprising amount that must be done in order to determine how muscles work. The research presented in this thesis focuses on an understudied portion of muscle function, water transport across sarcoplasmic reticulum. More precisely, the effects of gold nanoparticles on water transport across skeletal muscle sarcoplasmic reticulum. A background will first be presented in order to set a comprehensible framework for the research question and methodology.

\section{General Background of Muscle Function}

Mammalian muscle tissue is divided into three subtypes: skeletal, cardiac, and smooth muscle. Each type has features common to all muscle, although each have aspects unique to their subtype. Muscle contraction is elicited by a nerve impulse. This nerve impulse depolarizes a membrane called the sarcolemma, the surface membrane of the muscle cell. There are invaginations on the sarcolemma, called T-tubules, which conduct the depolarization to all parts of the cell simultaneously. The process that links depolarization of the surface membrane and the contraction of the cell is referred to as excitation-contraction coupling.

The T-tubule membrane contains the dihydropyridine receptor. When this transmembrane protein is activated by a change in voltage, it triggers the opening of the ryanodine receptor (RyR) in the membrane of the sarcoplasmic reticulum (SR). The SR is the storage site of calcium ions that are released upon the opening of the RyR (Alberts, 2015).

In order to properly understand how gold nanoparticles may be behaving with respect to $\mathrm{SR}$, it is important to describe the redox properties of both the nanoparticle and SR. 


\section{$\underline{\text { Redox Potential }}$}

Much research has indicated that the redox state of the muscle cell significantly influences the functioning of the calcium release channel (Eu et al, 2000; Feng et al, 2000; Xia et al, 2000). A more reduced cellular environment encourages the closing of the RyR, while a more oxidized cellular environment encourages the opening of the receptor. It has been established that the oxidation of sulfhydryl groups associated with the RyR activate its release mechanism, whereas the reduction of these thiol groups close down the channel (Xia et al, 2000). This information may be significant to the experiments that follow because the gold nanoparticles that are used demonstrate redox properties.

A gold nanoparticle (AuNP) capped with mercaptocarborane ligands is used in this study. This nanoparticle demonstrates that it is susceptible to further reduction and suffers reductive loss of its thiolate ligands (Ciorin et al, 2011).

\section{Water Transport}

Previous research on water transport across SR is little to nonexistent. Unfortunately, the same can be said for SR-nanoparticle interactions. Research involving AuNPs has shown that their physical and chemical properties may make them suitable for several cellular and medical applications (Giljohann, 2010). Many of the studies that use AuNPs have been interested in therapeutic topics such as gene-regulating agents, drug carriers, and imaging agents. The goal of this research is to shed some light on how AuNPs influence water transport across SR and to determine the molecular mechanism underlying this process. 


\section{Materials and Methods:}

A light scattering methodology was used in order to investigate water transport across SR. Vesicles of SR were equilibrated in a hypotonic solution (5 mM HEPES-KOH, pH 7.0) which causes them to swell. When solutes are added making the solution less hypotonic, water leaves the vesicles causing them to shrink. Changes in vesicle size were monitored with a fluorimeter by measuring light scattering at right angles at a wavelength of $400 \mathrm{~nm}$. Shrinkage of the vesicles corresponds to an increase in scattering (at $90^{\circ}$ ) and swelling corresponds to a decrease.

All experiments were done in a $3 \mathrm{~mL}$ quartz cuvette. The $5 \mathrm{mM}$ HEPES buffer, adjusted to $\mathrm{pH} 7$ with $\mathrm{KOH}$, was used in each experiment. A $0.15 \mathrm{mg} / \mathrm{ml}$ concentration of SR vesicles was also used in each experiment. AuNP concentrations of $200 \mathrm{nM}$ and $600 \mathrm{nM}$ were used depending on the trial. Liposome trials were also carried out in order to observe the behavior of phospholipid vesicles in the presence of AuNPs. A $0.15 \mathrm{mg} / \mathrm{ml}$ concentration of 1-palmitoyl-2oleoyl-sn-glycero-3-phosphocholine (POPC) vesicles (which mimics mammalian lipid composition) was used in these experiments. These phospholipid vesicle experiments also used AuNP at concentrations of $200 \mathrm{nM}$ and $600 \mathrm{nM}$. Another type of phospholipid vesicle composed of asolectin was also examined. The same methods and materials described above were used in all vesicle experiments. Every trial used a $20 \mathrm{mM}$ addition of sucrose as the solute to make the solution less hypotonic causing the vesicles to shrink.

For all water transport studies, sarcoplasmic reticulum vesicles were prepared from rabbit hind leg and back white skeletal muscles according to the method of Favero and Abramson (Favero, 1994). All asolectin vesicles (Sigma product number D00135349) used for the water transport experiments were also prepared using methods described by Favero and Abramson. 
POPC vesicles were prepared using a LUVET method, a procedure developed and provided by Avanti Polar Lipids, Inc. ("Preparing Large, Unilamellar Vesicles", n.d.). The mercaptoboranecapped gold nanoparticles used in this research were prepared according to the method of Ciorin and colleagues (Ciorin et al, 2011). The AuNPs were a gift of Professor Mathias Brust, University of Liverpool, England.

The extruder used in the LUVET method is an Avanti Polar Lipids, Inc. extruder set with holder/heating block. The extruder enabled us to generate a uniform mixture of unilamellar liposomes of diameter $200 \mathrm{~nm}$. The fluorimeter used in this research is the Hitachi F-4500 Fluorescence Spectrophotometer. 


\section{Data Analysis:}

\section{SR w/ 600 n m A N P}

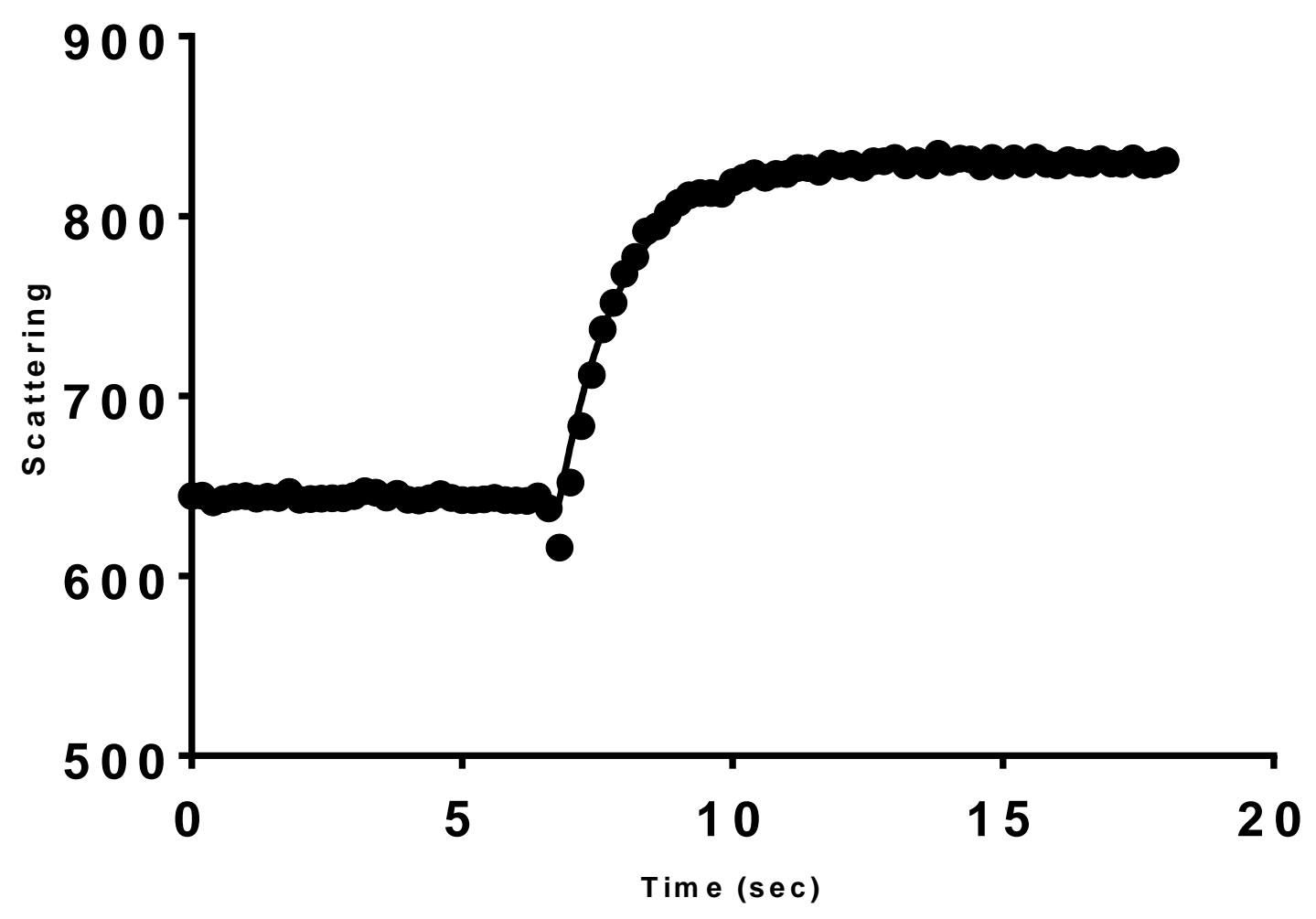

Figure 1: Illustrated here is fluorimetric data from an assay containing a $0.15 \mathrm{mg} / \mathrm{ml}$ concentration of SR in $5 \mathrm{mM}$ HEPES-KOH buffer. This trial additionally contained a $600 \mathrm{nM}$ concentration of AuNPs. This concentration can vary depending on the trial. At $\mathrm{t}=\sim 7 \mathrm{sec}, 20$ $\mathrm{mM}$ sucrose is injected into the cuvette and the absorbance at $400 \mathrm{~nm}$ is recorded as a function of time.

The independent variable of figure 1 is time measured in seconds. The dependent variable is the measure of the amount of light scattered at an angle of $90^{\circ}$. Figure one can be broken into and viewed as three sections: The baseline, the jump, and the plateau. The baseline is the flat line 
that begins at $\mathrm{Y}_{\mathrm{o}}$ and ends right before a sudden rise in the $\mathrm{Y}$ value. The jump begins immediately after the baseline and ends right before the plateau. The plateau is the final Y value.

The purpose of having the baseline is to make sure the solution was at equilibrium as well as to make certain no noise, bubbles, or any other unaccounted variables were present. A relatively level initial Y value for 30 seconds indicated that the solution was isotonic and no debris was present in the fluorimeter or cuvette. The jump indicates the point when sucrose was added into the solution in order to make the solution less hypotonic and cause the SR vesicles to shrink. This caused an increase in the amount of scattering present at $90^{\circ}$. The solution eventually returns to equilibrium, or it plateaus, and adopts a new and final Y value present for the duration of the trial.

The following experiments were carried out: Three trials using SR vesicles with no AuNPs present; five trials using SR vesicles and $200 \mathrm{nM}$ AuNPs; four trials using SR vesicles and $600 \mathrm{nM}$ AuNPs; three trials using POPC vesicles, in the absence of AuNPs; four trials using POPC vesicles and $200 \mathrm{nM}$ AuNPs; four trials using POPC vesicles and $600 \mathrm{nM}$ AuNPs; two trials using asolectin vesicles in the absence of AuNPs; two trials using asolectin vesicles and 200 nM AuNPs; one trial using asolectin vesicles and 600 nM AuNPs.

After the experimental data were collected, all data were fit to the formula:

$$
y=Y 0+A\left(1-e^{-b t}\right)
$$

where $A$ is the change in scattering, $\mathrm{Y}_{\text {final }}-\mathrm{Y}_{\text {initial }}, b$ is the rate constant, and $t$ is time in seconds. The change in scattering is related to the amount of water released, which is how it will be referred to for the remainder of this thesis. 
The initial rate of water transport across the vesicles, expressed as the rate of change of the nonlinear regression formula at $\mathrm{t}=0$, was also calculated. The derived formula is simply written as follows:

$$
\text { Initial rate }=\mathrm{Ab}
$$

Data in each trial category pertinent to either the amount of water released, $A$, the rate constant, $b$, or the initial rate of water transport across the vesicles, $A b$, were averaged. These data were then graphed in order to observe if there were any statistically significant disparities between the three kinds of vesicles in relation to these three formula components. Figures 2, 3, and 4 in the results section show these comparisons. 


\section{Results:}

The following experiments were carried out with three types of vesicle preparations sarcoplasmic reticulum vesicles (SR), asolectin vesicles (Asolectin), and POPC vesicles (POPC). The reason for carrying out these three types of experiments is to understand whether the water transport properties observed with sarcoplasmic reticulum vesicles are due to a specific protein found in the SR or whether it is due to the water permeability of the lipid membrane.

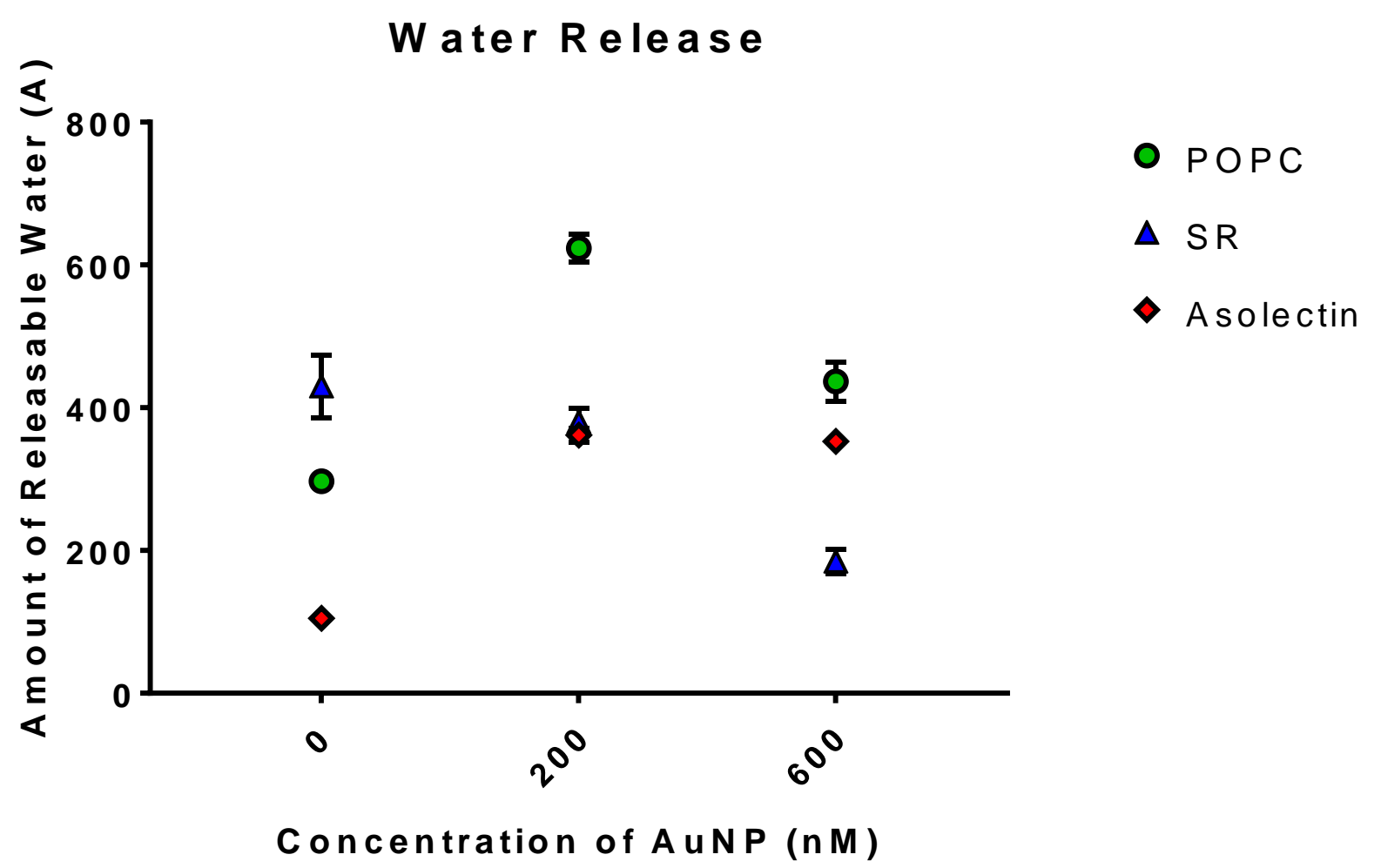

Figure 2: The total amount of water released from vesicles with varying concentrations of gold nanoparticles $(0,200$, and $600 \mathrm{nM})$ immediately after the addition of $20 \mathrm{mM}$ of sucrose into the solution. 


\section{Rate Constant}

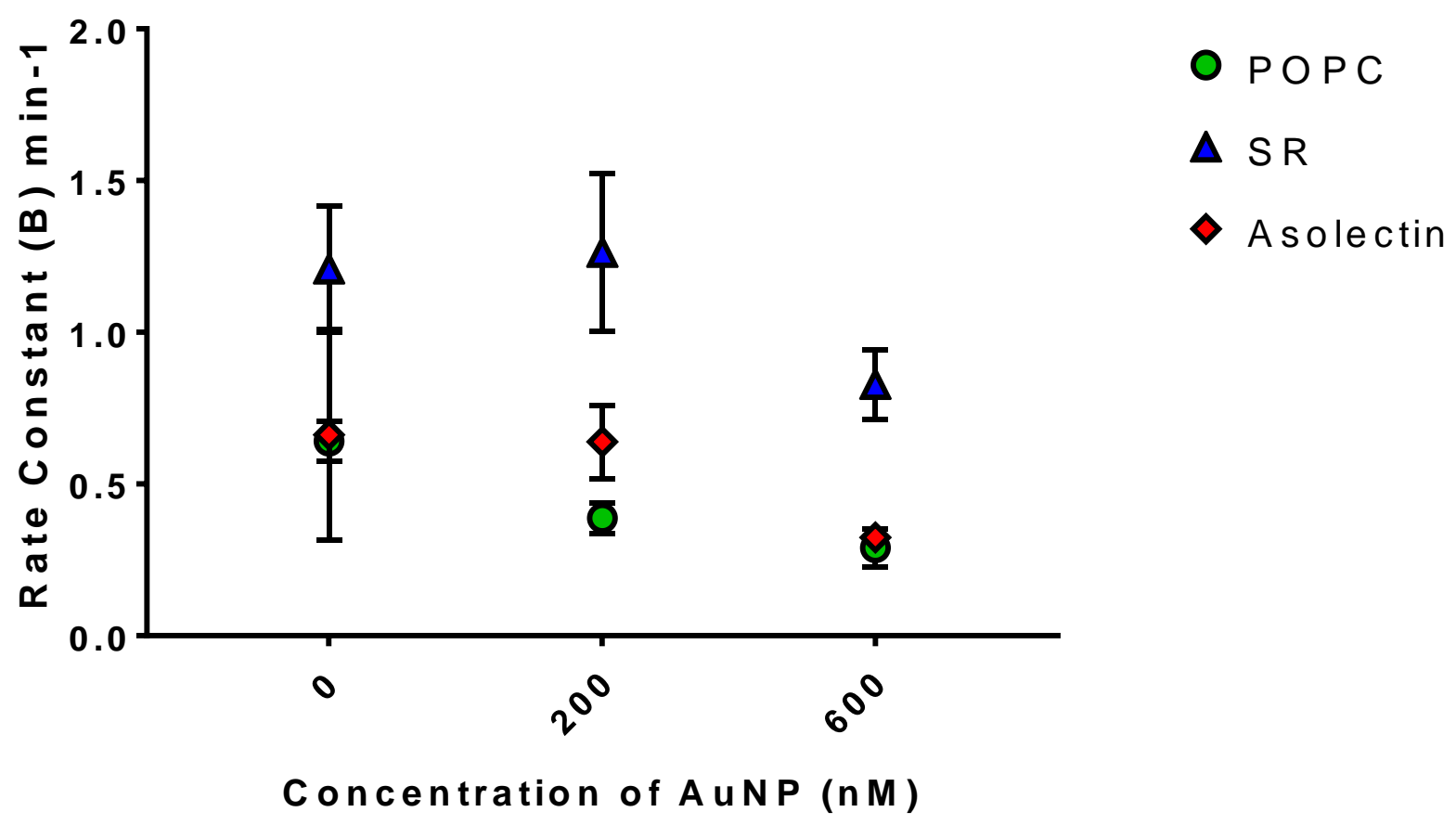

Figure 3: The calculated rate constant of water transport across vesicles with varying concentrations of gold nanoparticles $(0,200$, and $600 \mathrm{nM})$ immediately after the addition of 20 $\mathrm{mM}$ of sucrose added to the solution. 


\section{Rate of Water Transport}

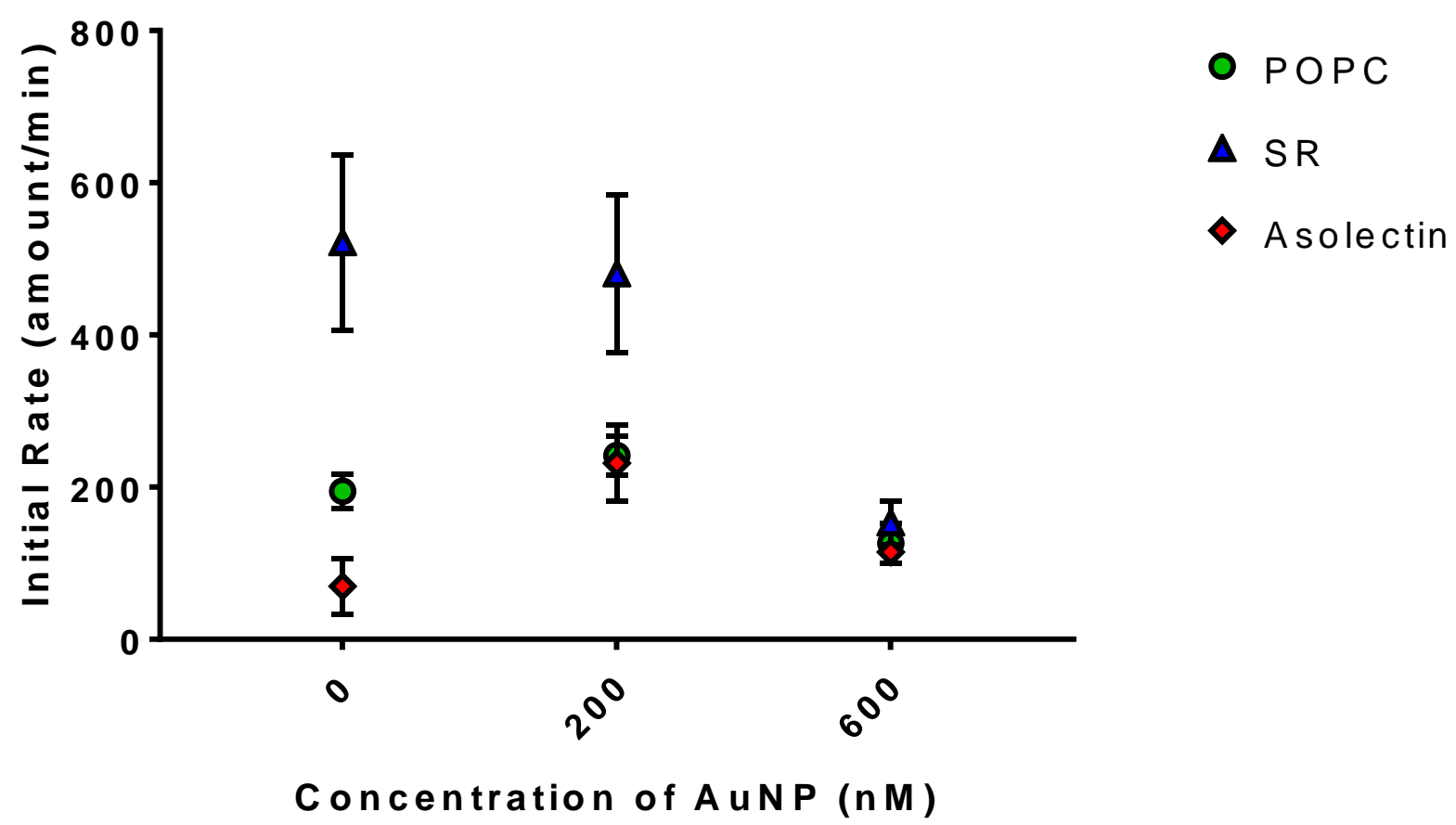

Figure 4: The calculated initial rate of water transport across the vesicles with varying concentrations of gold nanoparticles (0, 200, and 600 nanomolar) immediately after the addition of $20 \mathrm{mM}$ of sucrose into the solution. 


\section{Discussion:}

The experimental data indicate that the observed water transport properties of the sarcoplasmic reticulum is not due to the proteins present in the plasma membrane, but rather due to the water permeability of the lipid membrane. While there are noticeable differences in the figures that contrast sarcoplasmic reticulum and liposomes, the differences are only about two or three times in size and not by an order of magnitude. In other words, the differences seen between these two groups are relatively small. These differences likely stem from the fact that there are proteins present in the lipid membrane of sarcoplasmic reticulum. It must also be taken into account that the membrane composition of sarcoplasmic reticulum is not as well-defined and homogeneous as the membrane composition of the asolectin and POPC vesicles. In rabbit back and hind leg muscles, sarcoplasmic reticulum contains approximately $4 \%$ phosphatidylinositol, 5\% sphingomyelin, $68 \%$ phosphatidylcholine and a phospholipid/cholesterol molar ratio of 7.7 (Borchman, 1982).

Before the liposome experiments were conducted, it was believed that nanoparticles affected the proteins present on SR vesicles, which was responsible the rapid rate of water transport across the SR membrane. It was thought that aquaporins existed on the SR and that AuNPs somehow altered their behavior. Data from POPC and asolectin vesicles now suggest that differing concentrations of AuNPs alter the structure of the lipid membrane in order to make it more or less permeable to water transport. Aquaporins are likely not present on SR because of its shared similarities with POPC and asolectin vesicles.

In summary, the effects of AuNPs on water transport across SR suggest a decrease in the rate of water transport, a decrease in the amount of water released, and a decrease in the rate constant of water transport across SR vesicles as the concentration of AuNPs increase. 
AuNPs likely produce this effect by altering the behavior of the lipids on the sarcoplasmic reticulum plasma membrane, causing them to be less permeable to water transport. The mechanism behind this isn't yet known and would be an interesting subject to investigate further after this thesis. 


\section{Acknowledgments:}

I would like to give my honest and wholehearted thanks to the following people that made this thesis possible:

First and foremost my advisor, Dr. Jonathon Abramson, for allowing me to be a part of his lab, helping me develop this project, and supporting me throughout the entire thesis process. He has taught me a lot about how to be a proper scientist, and since he helped me produce this thesis I have a degree now too. That's pretty cool.

Dr. Drake Mitchell for his help in preparing the POPC vesicles used in this thesis. Those liposomes helped me shed light on how AuNPs affected SR.

Rob Klipp for his expert guidance and knowledge of the lab. Whenever something seemed lost, he always knew where it was. That sheep cardiac muscle preparation was an incredible thing to see too.

Jack for his company and good sense of humor while in the lab.

Mathias and his colleagues for supplying our lab with an ample amount of AuNPs.

And last, but not least, my family. My sister for her non-stop love and support throughout my college career. My niece for her happy and goofy nature, reminding me of the right way to approach life (with a smile!). My mom for her unconditional love and the sacrifices she made so I can be where I am now, and always making sure I'm well fed. My dad for always knowing how to make me laugh, always being the proudest of me in the family, and making me the hardworking and diligent man I am today. 


\section{Bibliography}

Alberts, B., Johnson, A., Lewis, J. H., and Morgan, D. (2015). Molecular Biology of the Cell, New York, NY: Garland Science.

Borchman, Douglas, Robert Simon, and Ellen Bicknell-Brown. "Variation in the lipid composition of rabbit muscle sarcoplasmic reticulum membrane with muscle type." Journal of Biological Chemistry 257.23 (1982): 14136-14139.

Cioran, Ana M., et al. "Mercaptocarborane-capped gold nanoparticles: electron pools and ion traps with switchable hydrophilicity." Journal of the American Chemical Society 134.1 (2011): 212-221.

Eu, Jerry P., et al. "The skeletal muscle calcium release channel: coupled O2 sensor and NO signaling functions." Cell 102.4 (2000): 499-509.

Favero, TG, and JJ Abramson. "Thapsigargin-induced $\mathrm{Ca} 2$ release from sarcoplasmic reticulum and asolectin vesicles." Cell Calcium (1994): 183-89. Print.

Feng, Wei, et al. "Transmembrane redox sensor of ryanodine receptor complex." Journal of Biological Chemistry 275.46 (2000): 35902-35907.

Giljohann, David A., et al. "Gold nanoparticles for biology and medicine." Angewandte Chemie International Edition 49.19 (2010): 3280-3294.

"Preparing Large, Unilamellar Vesicles by Extrusion (LUVET)." Avanti Polar Lipids. Avanti Polar Lipids, Inc., n.d. Web. 26 May 2017. 
Xia, Ruohong, Thomas Stangler, and Jonathan J. Abramson. "Skeletal muscle ryanodine receptor is a redox sensor with a well-defined redox potential that is sensitive to channel modulators." Journal of Biological Chemistry 275.47 (2000): 36556-36561. 